\title{
BANACH SUBSPACES OF SPACES OF HOLOMORPHIC FUNCTIONS AND RELATED TOPICS
}

\author{
VERÓNICA DIMANT AND SEÁN DINEEN
}

\section{§1. Introduction.}

Our initial interest in the topics discussed in this article was motivated by a desire to extend to homogeneous polynomials the following result of Dimant and Zalduendo [13];

(1.1) if the Banach space E has a shrinking unconditional Schauder basis then $c_{0} \nrightarrow \mathscr{L}\left({ }^{n} E\right)$ if and only if the monomials, with the square ordering, form a basis for $\mathscr{L}\left({ }^{n} E\right)\left(\mathscr{L}\left({ }^{n} E\right)\right.$ is the space of continuous $n$-linear forms on $\left.E\right)$.

This is one of many results (see the references quoted in $\$ 4$ and §5) which have appeared in recent years dealing with the linear and geometric properties of the space of $n$-linear forms and homogeneous polynomials on a $\mathrm{Ba}$ nach space. The approach in many of these papers has followed the classical methods of functional analysis. Indeed, following this approach and using results in [9] and [10] yields, fairly rapidly, a generalization of (1.1) to polynomials on stable Banach spaces (a Banach space is stable if $E \cong E \times E$ ). However, a recent result of Díaz [11] indicates that the same approach is not suitable for non-stable Banach spaces. Our approach was more function theoretic, in that we treated polynomials as functions on a set rather than as points in a Banach space. This broadened the scope of our investigation, led to a closer examination of each of the concepts involved in (1.1), yielded results which unified those of previous authors and, at the same time, revealed the natural setting for certain constructions.

We extended our investigation of the condition " $c_{0} \hookrightarrow \mathscr{P}\left({ }^{n} E\right)$ " to "c $c_{0} \nrightarrow \mathscr{H}(\mathscr{U})$ ". This prompted a look at the general question of lifting results from spaces of homogeneous polynomials to spaces of holomorphic functions. Since this topic has only been briefly touched upon in the literature we discuss it in $\S 2$. In $\S 3$ we return and solve the lifting problem asso- 
ciated with "c$c_{0} \hookrightarrow \mathscr{H}(\mathscr{U})$ ". To illustrate the results in $\S 3$ we looked at the monomial basis with the square order. Our function theoretic approach uncovered finite dimensional decompositions as a more natural object of study and we discuss these in $\S 4$. In this section we also considered the following result of Alencar [1] which may be readily compared with (1.1);

(1.2) if $E$ is a reflexive Banach space with a Schauder basis then $\mathscr{P}\left({ }^{n} E\right)$ is reflexive if and only if the monomials, with the square order, are a Schauder basis for $\mathscr{P}\left({ }^{n} E\right)\left(\mathscr{P}\left({ }^{n} E\right)\right.$ is the space of continuous $n$-homogeneous polynomials on $E)$.

We generalise and obtain an independent proof of this result. Finally, on comparing once more (1.1) and (1.2) we found that we can bridge the gap between the conditions of non-containment of $c_{0}$ and reflexivity, by means of an unconditionality hypothesis on the Schauder basis. This is discussed in $§ 5$. We refer to $[12,24]$ for Banach space theory and to $[14,16]$ for infinite dimensional holomorphy.

\section{§2. Lifting results from homogeneous polynomials to holomorphic functions.}

In this section we discuss the general problem of lifting results from spaces of homogeneous polynomials to spaces of holomorphic functions on balanced domains. Spaces of homogeneous polynomials normally lie within a more manageable collection than the corresponding space of holomorphic functions. For instance, the space of C-valued continuous $n$-homogeneous polynomials on a Banach space $E, \mathscr{P}\left({ }^{n} E\right)$, endowed with the strong topology, is a Banach space, while the space of C-valued holomorphic functions on $E, \mathscr{H}(E)$, endowed with any of its natural topologies is never a Banach space and, indeed, only a Frechet space when $E$ is finite dimensional (and in this case it is even a Fréchet-nuclear space). Results for $\mathscr{H}(E)$ which only require the result for some subspace of $\mathscr{H}(E)$ are often immediate from the polynomial results but results which depend on all subspaces of $\mathscr{H}(E)$ generally require further analysis.

For example, if $\ell_{\infty} \hookrightarrow \mathscr{P}\left({ }^{n} E\right)$ for some positive integer $n$ then we see immediately that $\ell_{\infty} \hookrightarrow\left(\mathscr{H}(E), \tau_{\omega}\right)$, while $\mathscr{P}\left({ }^{n} E\right)$ reflexive for all $n$ implies $\left(\mathscr{H}(E), \tau_{\omega}\right)$ is reflexive if $E$ is separable and has the approximation property but this latter result on reflexivity is not at all immediate ([14]). Another non- trivial example is given in [3] where it is shown, for $E$ Frechet-Montel, that $\tau_{0}=\tau_{\omega}$ on $\mathscr{H}(E)$ if and only if $\tau_{0}=\tau_{\omega}$ on $\mathscr{P}\left({ }^{n} E\right)$ for all $n$.

In this article we consider collections of holomorphic functions on a balanced domain in a Banach space. In this case the space of holomorphic functions has a Schauder decomposition into a sequence of Banach spaces, 
e.g. $\left(\mathscr{H}(E), \tau_{\omega}\right)$ has $\left\{\left(\mathscr{P}\left({ }^{n} E\right),\|\cdot\|\right)\right\}_{n=0}^{\infty}$ as a Schauder decomposition. This decomposition identifies a sequence of "priviliged" Banach subspaces of $\left(\mathscr{H}(E), \tau_{\omega}\right)$. Finite sums, quotients, subspaces and the range by linear isomorphisms (of $\left(\mathscr{H}(E), \tau_{\omega}\right)$ ) of $\mathscr{P}\left({ }^{n} E\right)$ yield further Banach subspaces of $\left(\mathscr{H}(E), \tau_{\omega}\right)$. Are these "essentially" all the Banach subspaces of $\left(\mathscr{H}(E), \tau_{\omega}\right)$ ? A slightly less general and more precise way of looking at this is as follows:

Let $(T)$ denote a certain property of Banach spaces (e.g. reflexivity, weak sequential compactness, the Dunford-Pettis property). If $\left(\mathscr{P}\left({ }^{n} E\right),\|\cdot\|\right)$ has $(T)$ for all $n$, does every Banach subspace of $\left(\mathscr{H}(E), \tau_{\omega}\right)$ have property $(T)$ ?

Another variation of this question can be posed in the following way: if $F$ is a Banach subspace of $\left(\mathscr{H}(E), \tau_{\omega}\right)$ and $\left(x_{j}\right)_{j}$ is a basic sequence in $F$, does there exists a subsequence $\left(y_{j}\right)_{j}$ of $\left(x_{j}\right)_{j}$, and a positive integer $n$ such that $\left(y_{j}\right)_{j}$ is equivalent to a basic sequence in $\left(\mathscr{P}\left({ }^{n} E\right),\|\cdot\|\right)$ ?

The same problems can clearly be posed for different topologies and other spaces of holomorphic functions and it is also obvious how the same questions may be phrased for $\mathscr{S}$-absolute decompositions. We now give two examples which show that the general problems outlined above are reasonable.

Example 1 . Let $E$ denote a Banach space and let $\left(n_{j}\right)_{j}$ denote a strictly increasing sequence of positive integers. For each $j$ let $P_{j}$ denote a non-zero element of $\mathscr{P}\left({ }^{n_{j}} E\right)$. Let $F$ denote the closed subspace of $\left(\mathscr{H}(E), \tau_{\omega}\right)$ generated by $\left(P_{j}\right)_{j}$. Taylor series expansions at the origin show easily that $\left(P_{j}\right)_{j}$ is a basis for $F$. Hence if $f \in F$ then $f=\sum_{j=0}^{\infty} \beta_{j} P_{j}$ for some sequence of scalars $\left(\beta_{j}\right)_{j}$.

Now the $\tau_{\omega}$ topology on $\mathscr{H}(E)$ is generated by the seminorms

$$
p(f)=\sum_{n=0}^{\infty}\left\|\frac{\widehat{d}^{n} f(0)}{n !}\right\|_{K+\alpha_{n} B}
$$

for $f=\sum_{n=0}^{\infty} \frac{\widehat{d^{n}} f(0)}{n !} \in \mathscr{H}(E)$, where $B$ is the open unit ball of $E, K$ is a compact subset of $E$ and $\left(\alpha_{n}\right)_{n} \in c_{0}$.

Hence

$$
p\left(\sum_{j=0}^{\infty} \beta_{j} P_{j}\right)=\sum_{j=0}^{\infty}\left|\beta_{j}\right|\left\|P_{j}\right\|_{K+\alpha_{n_{j}} B}
$$

and $\left(P_{j}\right)_{j}$ is an absolute basis for $F$ with weights $\left(\left\|P_{j}\right\|_{K+\alpha_{n_{j} B} B}\right)_{j}$ where $K$ is compact in $E$ and $\left(\alpha_{n_{j}}\right)_{j} \in c_{0}$. In particular, we see that $\left(\left\|P_{j}\right\|_{2 K+2 \alpha_{n_{j} B} B}\right)_{j}=\left(2^{n_{j}}\left\|P_{j}\right\|_{K+\alpha_{n_{j}} B}\right)_{j}$ is also a weight and, since $\sum_{j} \frac{1}{2^{n_{j}}}<\infty$, the Grothendieck-Pietsch criterion implies that $F$ is a nuclear subspace of $\left(\mathscr{H}(E), \tau_{\omega}\right)$. The nuclear space $F$, which is generated by homogeneous poly- 
nomials all of different degrees, may be regarded as the extreme example of a space not contained in $\mathscr{P}\left({ }^{n} E\right)$ for any $n$.

EXAmple 2. Let $\left(P_{m}\right)_{m=n+1}^{\infty}$ denote a basic sequence of unit vectors in $\mathscr{P}\left({ }^{n} E\right), n$ a positive integer and let $\left(Q_{m}\right)_{m=n+1}^{\infty}$ denote a sequence of continuous polynomials, $Q_{m}$ being $m$-homogeneous. We suppose $\left\|Q_{m}\right\|_{B}^{1 / m} \underset{m \rightarrow \infty}{\longrightarrow} 0$, where $B$ is the unit ball of $E$. Let $F$ denote the (Banach) subspace of $\mathscr{P}\left({ }^{n} E\right)$ generated by $\left(P_{m}\right)_{m=n+1}^{\infty}$ and let $G$ denote the closed subspace of $\left(\mathscr{H}(E), \tau_{\omega}\right)$ generated by $\left(P_{m}+Q_{m}\right)_{m=n+1}^{\infty}$. We claim that $F$ and $G$ are isomorphic as locally convex spaces and hence $G$ is also a Banach subspace of $\left(\mathscr{H}(E), \tau_{\omega}\right)$.

$$
\begin{aligned}
& \text { If } f=\sum_{j=0}^{\infty} \frac{\widehat{d}^{j} f(0)}{j !} \in G \text { then it is easily seen that } \\
& \frac{\widehat{d^{j}} f(0)}{j !}= \begin{cases}0 & \text { if } j<n \\
\sum_{m=n+1}^{\infty} \beta_{m} P_{m} & \text { if } j=n \\
\beta_{j} Q_{j} & \text { if } j>n .\end{cases}
\end{aligned}
$$

Since the mapping $f \in\left(\mathscr{H}(E), \tau_{\omega}\right) \mapsto \frac{\widehat{d}^{n} f(0)}{n !} \in\left(\mathscr{P}\left({ }^{n} E\right),\|\cdot\|\right)$ is continuous it follows that the mapping

$$
J:=\sum_{m=n+1}^{\infty} \beta_{m}\left(P_{m}+Q_{m}\right) \in G \longmapsto \sum_{m=n+1}^{\infty} \beta_{m} P_{m} \in F
$$

is continuous. It is also immediate that $J$ is a bijection.

Let

$$
R_{j}:=\sum_{m=n+1}^{\infty} \beta_{m}^{j} P_{m} \in F \longrightarrow 0 \text { as } j \rightarrow \infty .
$$

Since $\left(P_{m}\right)_{m}$ is a basic sequence of unit vectors in a Banach space it follows that

$$
\sup _{m, j}\left|\beta_{m}^{j}\right|<\infty
$$

and

$$
\beta_{m}^{j} \underset{j \rightarrow \infty}{\longrightarrow} \quad \forall m>n .
$$

Hence $\sup _{j, m}\left\|\beta_{m}^{j} Q_{m}\right\|_{r B}<\infty$ for all $r>0$ and, by (2.1), 


$$
\left\{J^{-1}\left(R_{j}\right)=\sum_{m=n+1}^{\infty} \beta_{m}^{j} P_{m}+\sum_{m=n+1}^{\infty} \beta_{m}^{j} Q_{m}\right\}_{j}
$$

is a bounded sequence in $\left(\mathscr{H}(E), \tau_{\omega}\right)$.

Since a bounded sequence $\left(f_{j}\right)_{j}$ in $\left(\mathscr{H}(E), \tau_{\omega}\right)$ is a null sequence if and only if $\frac{\widehat{d}^{k} f_{j}(0)}{k !}$ is a null sequence in $\mathscr{P}\left({ }^{k} E\right)$ for all $k$ [14, lemma 3.28], it follows, by (2.2), that $\left(J^{-1}\left(R_{j}\right)\right)_{j}$ is a null sequence in $\left(\mathscr{H}(E), \tau_{\omega}\right)$. Hence $J^{-1}$ is continuous and $J$ is a linear isomorphism from $G$ onto $F$ and $G$ is a Banach subspace of $\left(\mathscr{H}(E), \tau_{\omega}\right)$.

The Banach space $G$ is not one of the "essential" Banach spaces we mentioned earlier but was obtained by perturbing an essential Banach space. In this case what might be deemed the nuclear perturbation was absorbed into the more dominant Banach space structure to produce a Banach space. More refined examples of this type may suggest ways of constructing Banach spaces which are not essential or at least point to the middle zone between nuclear and Banach spaces where counterexamples might lie.

\section{§3. Function Spaces containing $c_{0}$.}

In this section we give a positive solution to the lifting problem for the property "containment of $c_{0}$ ". In order to obtain the same result for different spaces and topologies, e.g. holomorphic germs and holomorphic functions of bounded type, we prove an abstract result using $\mathscr{S}$-absolute decompositions. Let $\mathscr{S}=\left\{\left(\alpha_{n}\right)_{n}: \alpha_{n} \in \mathrm{C}\right.$ and $\left.\limsup _{n \rightarrow \infty}\left|\alpha_{n}\right|^{1 / n} \leq 1\right\}$.

Definition 3. A decomposition $\left\{E_{n}\right\}_{n}$ of a locally convex space $E$ is an $\mathscr{S}$-absolute decomposition if

(a) for each $\left(\alpha_{n}\right)_{n} \in \mathscr{S}$ and each $x=\sum_{n=1}^{\infty} x_{n} \in E, \quad x_{n} \in E_{n} \quad \forall n$, we have that $\sum_{n=1}^{\infty} \alpha_{n} x_{n} \in E$.

(b) if $p$ is a continuous semi-norm on $E$ and $\left(\alpha_{n}\right)_{n} \in \mathscr{S}$, then the seminorm

$$
q\left(\sum_{n=1}^{\infty} x_{n}\right):=\sum_{n=1}^{\infty}\left|\alpha_{n}\right| p\left(x_{n}\right), \quad \text { for } x=\sum_{n=1}^{\infty} x_{n} \in E, x_{n} \in E_{n} \forall n
$$

is continuous.

THEOREM 4. Let $\left\{E_{n}\right\}_{n}$ denote an $\mathscr{S}$-absolute decomposition of the locally convex space $E$ and suppose each $E_{n}$ is a Banach space. If the sequence $\left\{x_{m}=\sum_{n=1}^{\infty} x_{n, m}\right\}_{m=1}^{\infty}$, where $x_{n, m} \in E_{n} \forall n, m$, is equivalent to the unit vector basis of $c_{0}$, then for each integer $n$ either 
(a) $\left(x_{n, m}\right)_{m=1}^{\infty}$ is a null sequence in $E_{n}$, or

(b) there exists a strictly increasing sequence of positive integers $\left(m_{j}\right)_{j}$ such that the sequence $\left(x_{n, m_{2 j+1}}-x_{n, m_{2 j}}\right)_{j}$ is equivalent to the unit vector basis of $c_{0}$.

Moreover, (b) occurs for some positive integer $n$ and hence $c_{0} \hookrightarrow E$ if and only if there exists a positive integer $n$ such that $c_{0} \hookrightarrow E_{n}$.

Proof. Fix a positive integer $n$ and let $\|\cdot\|_{n}$ denote the norm on the Banach space $E_{n}$. Let $\left\|\sum_{m=1}^{\infty} \lambda_{m} x_{m}\right\|_{0}=\sup _{m}\left|\lambda_{m}\right|$.

By our hypothesis there exists $C:=C(n)$ such that for any sequence of scalars $\left(\alpha_{m}\right)_{m}$ and any positive integer $k$

$$
\left\|\sum_{m=1}^{k} \alpha_{m} x_{n, m}\right\|_{n} \leq C\left\|\sum_{m=1}^{k} \alpha_{m} x_{m}\right\|_{0}=C \sup _{m \leq k}\left|\alpha_{m}\right| .
$$

This implies in particular that $\left(x_{n, m}\right)_{m=1}^{\infty}$ is a bounded sequence in $\left(E_{n},\|\cdot\|_{n}\right)$.

We first claim that $\left(x_{n, m}\right)_{m=1}^{\infty}$ does not contain a subsequence $\left(x_{n, m_{j}}\right)_{j=1}^{\infty}$ equivalent to the unit vector basis of $\ell_{1}$. Otherwise, there would exist $C_{1}>0$ such that

$$
C_{1} \sum_{j=1}^{k}\left|\alpha_{j}\right| \leq\left\|\sum_{j=1}^{k} \alpha_{j} x_{n, m_{j}}\right\|_{n}
$$

for any sequence of scalars $\left(\alpha_{j}\right)_{j}$ and any positive integer $k$. This contradicts (3.1) for large $k$ and establishes our claim.

By Rosenthal's $\ell_{1}$ theorem [12, p. 201] or [24, p. 99] it follows that every subsequence of $\left(x_{n, m}\right)_{m=1}^{\infty}$ contains a weak Cauchy subsequence. Let $\left(x_{n, m_{i}}\right)_{i=1}^{\infty}$ denote a weak Cauchy subsequence of $\left(x_{n, m}\right)_{m=1}^{\infty}$. We suppose that $\left(x_{n, m_{i}}\right)_{i=1}^{\infty}$ is not a norm Cauchy sequence. By taking a subsequence, if necessary, we may suppose that there exists $\delta>0$ such that

$$
\left\|x_{n, m_{2 i+1}}-x_{n, m_{2 i}}\right\|_{n} \geq \delta \quad \text { for all } i \text {. }
$$

Since $\left(x_{n, m_{i}}\right)_{i=1}^{\infty}$ is a weak Cauchy sequence it follows that $\left(x_{n, m_{2 i+1}}-x_{n, m_{2 i}}\right)_{i}$ is a weakly null sequence and by the Bessaga-Pelczynski selection principle $[12$, p.42] or [24, p.7] we may suppose, again by taking a subsequence, that it is a basic sequence in $E_{n}$. For any sequence of scalars, $\left(\beta_{i}\right)_{i}$, and any positive integer $k$ we have by (3.1)

$$
\left\|\sum_{i=1}^{k} \beta_{i}\left(x_{n, m_{2 i+1}}-x_{n, m_{2 i}}\right)\right\|_{n} \leq C \sup _{i \leq k}\left|\beta_{i}\right| .
$$

Hence $\left(x_{n, m_{2 i+1}}-x_{n, m_{2 i}}\right)_{i}$ is equivalent to the unit vector basis of $c_{0}$ and (b) is satisfied by the sequence $\left(x_{n, m_{i}}\right)_{i=1}^{\infty}$. 
The remaining case is when every subsequence of $\left(x_{n, m}\right)_{m=1}^{\infty}$ contains a norm Cauchy subsequence. By choosing a subsequence if necessary we may suppose that there exists $x \in E_{n}$ such that

$$
\left\|x_{n, m}-x\right\|_{n} \leq 1 / 2^{m} \text { for all } m
$$

By (3.1),

$$
C \geq\left\|\sum_{m=1}^{k} x_{n, m}\right\|_{n} \geq k\|x\|_{n}-\sum_{m=1}^{k} \frac{1}{2^{m}}
$$

for all $k$. This implies $x=0$. Hence every subsequence of $\left(x_{n, m}\right)_{m=1}^{\infty}$ contains a norm null subsequence and we conclude that $\left(x_{n, m}\right)_{m=1}^{\infty}$ is a null sequence in $E_{n}$. This shows that the sequence $\left(x_{n, m}\right)_{m=1}^{\infty}$ satisfies (a) and we conclude that $\left(x_{n, m}\right)_{m=1}^{\infty}$ always satisfies either (a) or (b).

We now suppose that (a) is satisfied by $\left(x_{n, m}\right)_{m=1}^{\infty}$ for all $n$. Since $\left(x_{m}\right)_{m=1}^{\infty}$ is a bounded sequence in $E$, lemma 3.28 of [14] implies that $\left(x_{m}\right)_{m=1}^{\infty}$ is a null sequence in $E$. However, $\left(x_{m}\right)_{m=1}^{\infty}$ is equivalent to the unit vector basis of $c_{0}$ and this is impossible. Hence condition (b) is satisfied by some $n$. This completes the proof.

Corollary 5. If $E$ is a Banach space then $\left(\mathscr{P}\left({ }^{n} E\right),\|\cdot\|\right)$ contains $c_{0}$ for some positive integer $n$ if and only if the locally convex space $(\mathscr{H}, \tau)$ contains $c_{0}$, where $(\mathscr{H}, \tau)$ is any one of the following:

(1) $\mathscr{H}=\mathscr{H}(\mathscr{U}), \mathscr{U}$ balanced open in $E, \tau=\tau_{\omega}$ or $\tau_{\delta}$,

(2) $\mathscr{H}=\mathscr{H}(K), K$ compact balanced in $E$ and $\mathscr{H}(K)$ the space of holomorphic germs on $K, \tau=\tau_{\omega}$,

(3) $\mathscr{H}=\mathscr{H}_{b}(\mathscr{U}), \mathscr{U}$ balanced open in $E$, and $\mathscr{H}_{b}(\mathscr{U})$ the subspace of $\mathscr{H}(\mathscr{U})$ consisting of functions which are bounded on the bounded subsets of $\mathscr{U}$ which lie strictly inside $\mathscr{U}$ and $\tau$ the topology of uniform convergence on such sets.

Proof. For each of the spaces $(\mathscr{H}, \tau)$ the sequence $\left\{\left(\mathscr{P}\left({ }^{n} E\right),\|\cdot\|\right)\right\}_{n=0}^{\infty}$ is an $\mathscr{S}$-absolute decomposition [14, chapter 3] and it suffices to apply theorem 4.

Further examples of a similar kind can also be found by considering compact polynomials, weakly sequentially continuous polynomials, etc. It suffices to verify that lemma 3.28 of [14] applies in each particular case.

Since $\left(\mathscr{H}(\mathscr{U}), \tau_{\omega}\right)$ is not known to be a dual space the following corollary is also of interest.

COROLlary 6. If $\mathscr{U}$ is a balanced open subset of a Banach space then $c_{0} \hookrightarrow\left(\mathscr{H}(\mathscr{U}), \tau_{\omega}\right)$ if and only if $\ell_{\infty} \hookrightarrow\left(\mathscr{H}(\mathscr{U}), \tau_{\omega}\right)$. 
Proof. If $c_{0} \hookrightarrow\left(\mathscr{H}(\mathscr{U}), \tau_{\omega}\right)$ then corollary 5 implies that there exists a positive integer $n$ such that $c_{0} \hookrightarrow\left(\mathscr{P}\left({ }^{n} E\right),\|\cdot\|\right)$. Since $\left(\mathscr{P}\left({ }^{n} E\right),\|\cdot\|\right)$ is a dual Banach space it follows that $\ell_{\infty} \hookrightarrow\left(\mathscr{P}\left({ }^{n} E\right),\|\cdot\|\right) \hookrightarrow\left(\mathscr{H}(\mathscr{U}), \tau_{\omega}\right)$. Since $c_{0} \hookrightarrow \ell_{\infty}$ the converse is obvious and this completes the proof.

Example 7. (a) ([2]) If $T$ is Tsirelson's space ([24, p. 95]) then $\left(\mathscr{P}\left({ }^{n} T^{\prime}\right),\|\cdot\|\right)$ is reflexive for all $n$ hence $c_{0} \nrightarrow\left(\mathscr{H}(\mathscr{U}), \tau_{\omega}\right)$ for any balanced open subset $\mathscr{U}$ of $T^{\prime}$.

(b) By [4] (see also [5, example 7]), $\left(\mathscr{P}\left({ }^{n} c_{0}\right),\|\cdot\|\right)$ is a separable dual space for any positive integer $n$ and hence does not contain $c_{0}$. By theorem 4 , $\left(\mathscr{H}(\mathscr{U}), \tau_{\omega}\right)$ does not contain $c_{0}$ for any balanced open subset $\mathscr{U}$ of $c_{0}$.

(c) Let $T_{J}^{\prime}$ denote the James space modeled on the space $T^{\prime}$ of (a). By [5, proposition 15], $\left(\mathscr{P}\left({ }^{n} T_{J}^{\prime}\right),\|\cdot\|\right)$ has RNP for any positive integer $n$. Hence $c_{0} \nrightarrow\left(\mathscr{H}(\mathscr{U}), \tau_{\omega}\right)$ for any balanced open subset $\mathscr{U}$ of $T_{J}^{\prime}$.

\section{§4. Finite Dimensional Decompositions.}

We now examine the basis condition mentioned in (1.1) and (1.2). The following simple algebraic example motivated our move from Schauder basis to finite dimensional decompositions. We need the following notation.

If $m=\left(m_{1}, \ldots, m_{k}, 0, \ldots\right) \in \mathrm{N}^{(\mathrm{N})}$, we let $|m|=\sum_{i} m_{i}$ denote the degree of $m$ and $\ell(m)=\sup \left\{i: m_{i} \neq 0\right\}$, the length of $m$. We let $m !=m_{1} ! \ldots m_{k} !$.

Example 8 . Let $P$ denote an $n$-homogeneous polynomial on the space of all finite sequences of complex numbers. Then

$$
\begin{aligned}
P\left(\left(z_{i}\right)_{i=1}^{\infty}\right) & =\sum_{\substack{m \in \mathbb{N}(\mathbb{N}) \\
|m|=n}} a_{m} z^{m} ; \quad z^{m}=z_{1}^{m_{1}} \ldots z_{k}^{m_{k}}, \quad \ell(m)=k \\
& =\sum_{k=1}^{\infty}\left\{\sum_{\substack{|m|=n \\
\ell(m)=k}} a_{m} z^{m}\right\} \\
& =\sum_{k=1}^{\infty}\left\{P\left(\left(z_{i}\right)_{i=1}^{k}\right)-P\left(\left(z_{i}\right)_{i=1}^{k-1}\right)\right\} \\
& =a_{(n, 0, \ldots)} z_{1}^{n}+\sum_{\substack{n_{1}+n_{2}=n \\
n_{2}>0}} a_{\left(n_{1}, n_{2}, 0, \ldots\right)} z_{1}^{n_{1}} z_{2}^{n_{2}}+\cdots
\end{aligned}
$$

Note that since all the sequences are eventually zero, all the sums are finite and there is no difficulty with convergence.

We now develop this example topologically. We suppose that $E$ is a Banach space with Schauder decomposition $\left\{E_{j}\right\}_{j=1}^{\infty}$. An element $P \in \mathscr{P}\left({ }^{n} E\right)$ is called a monomial if there exists $m=\left(m_{i}\right)_{i} \in \mathrm{N}^{(\mathrm{N})}$ such that for all 
$\sum_{i=1}^{\infty} x_{i} \in E, x_{i} \in E_{i}$ for all $i$, and any sequence of scalars $\left(\lambda_{i}\right)_{i=1}^{\infty}$ we have

$$
P\left(\sum_{i=1}^{\infty} \lambda_{i} x_{i}\right)=\lambda_{1}^{m_{1}} \ldots \lambda_{k}^{m_{k}} P\left(\sum_{i=1}^{\infty} x_{i}\right)
$$

where $k=\ell(m)$. Clearly we must have $n=|m|$.

We denote by $\mathscr{P}_{k}\left({ }^{n} E\right)$ the space generated by

$$
\left\{P \in \mathscr{P}\left({ }^{n} E\right) ; P \text { is a monomial of degree } m, \ell(m)=k\right\} \text {. }
$$

If $P \in \mathscr{P}\left({ }^{n} E\right)$ and $m=\left(m_{i}\right)_{i} \in \mathbf{N}^{(\mathrm{N})}, \ell(m)=k$, we let

$$
P_{m}\left(\sum_{i=1}^{\infty} x_{i}\right)=\frac{|m| !}{m !} \frac{1}{(2 \pi i)^{k}} \int_{\left|\lambda_{1}\right|=1} \ldots \int_{\left|\lambda_{k}\right|=1} \frac{P\left(\sum_{i=1}^{k} \lambda_{i} x_{i}\right)}{\lambda_{1}^{m_{1}+1} \ldots \lambda_{k}^{m_{k}+1}} d \lambda_{1} \ldots d \lambda_{k} .
$$

We have $P_{m} \in \mathscr{P}_{\ell(m)}\left({ }^{n} E\right)$ and

$$
P(x)=\sum_{\substack{m \in \mathbb{N}(N) \\|m| n \mid=n}} P_{m}(x)
$$

for all $x$ in the algebraic span of $\left\{E_{j}\right\}_{j=1}^{\infty}$.

If $P \in \mathscr{P}\left({ }^{n} E\right)$ we let

$$
P_{(k)}=\sum_{\substack{m \in(N) \\ \ell(m)=k}} P_{m}
$$

Alternatively

$$
P_{(k)}\left(\sum_{i=1}^{\infty} x_{i}\right)=P\left(\sum_{i=1}^{k} x_{i}\right)-P\left(\sum_{i=1}^{k-1} x_{i}\right)
$$

for all $k$ and all $\sum_{i=1}^{\infty} x_{i} \in E$.

We let $\mathscr{P}_{\omega}\left({ }^{n} E\right)$ denote the subspace of $\mathscr{P}\left({ }^{n} E\right)$ consisting of all polynomials which are weakly continuous on bounded subsets of $E$. By [7] this coincides with the space of all $n$-homogeneous polynomials which are uniformly weakly continuous on bounded subsets of $E$ and if $E^{\prime}$ has the approximation property, and in particular if $E^{\prime}$ has a finite dimensional Schauder decomposition, then $\mathscr{P}_{\omega}\left({ }^{n} E\right)$ is the closed subspace of $\mathscr{P}\left({ }^{n} E\right)$ generated by $\left\{\varphi^{n}: \varphi \in E^{\prime}\right\}$.

If $\left\{E_{j}\right\}_{j}$ is a finite dimensional Schauder decomposition for the Banach space $E$ then $\mathscr{P}_{k}\left({ }^{n} E\right)$ is a finite dimensional space for all $k$ and $n$ and if, in addition, $\left\{E_{j}\right\}_{j}$ is shrinking then $\left\{E_{j}^{\prime}\right\}_{j}$ is a finite dimensional Schauder decomposition for $E^{\prime}$. 
In our next proposition we shall consider a Banach space with finite dimensional decomposition $\left\{E_{j}\right\}_{j}$. If $x \in E$ we will write $x=\sum_{j=1}^{\infty} x_{j}$ where $x_{j} \in E_{j}$ for all $j$ and we let $\Pi_{k}\left(\sum_{j=1}^{\infty} x_{j}\right)=\sum_{j=1}^{k} x_{j}$ for any positive integer $j$. The sequence $\left(\Pi_{k}\right)_{k=1}^{\infty}$ is an equicontinuous (or bounded) sequence of linear mappings and by renorming $E$ (if necessary) we can suppose that $\sup _{k}\left\|\Pi_{k}\right\|=1$. In such a case we say that the decomposition is monotone. We may assume without loss of generality that our decompositions are always monotone.

Proposition 9. If $\left\{E_{j}\right\}_{j}$ is a shrinking finite dimensional decomposition for the Banach space $E$ then, for any positive integer $n,\left\{\mathscr{P}_{k}\left({ }^{n} E\right)\right\}_{k=1}^{\infty}$ is a monotone finite dimensional decomposition for $\mathscr{P}_{\omega}\left({ }^{n} E\right)$.

Proof. Let $\left(P_{k}\right)_{k=1}^{\infty} \subset \mathscr{P}\left({ }^{n} E\right)$ with $P_{k} \in \mathscr{P}_{k}\left({ }^{n} E\right)$ for all $k$. Since

$$
\begin{aligned}
\left\|\sum_{i=1}^{k} P_{i}\right\| & =\sup _{\left\|\sum_{j=1}^{\infty} x_{j}\right\| \leq 1}\left|\sum_{i=1}^{k} P_{i}\left(\sum_{j=1}^{\infty} x_{j}\right)\right| \\
& =\sup _{\left\|\sum_{j=1}^{k} x_{j}\right\| \leq 1}\left|\sum_{i=1}^{k} P_{i}\left(\sum_{j=1}^{k} x_{j}\right)\right| \\
& =\sup _{\left\|\sum_{j=1}^{k} x_{j}\right\| \leq 1}\left|\sum_{i=1}^{k+1} P_{i}\left(\sum_{j=1}^{k} x_{j}\right)\right| \\
& \leq \sup _{\left\|\sum_{j=1}^{\infty} x_{j}\right\| \leq 1}\left|\sum_{i=1}^{k+1} P_{i}\left(\sum_{j=1}^{\infty} x_{j}\right)\right| \\
& =\left\|\sum_{i=1}^{k+1} P_{i}\right\|
\end{aligned}
$$

it follows that $\left\{\mathscr{P}_{k}\left({ }^{n} E\right)\right\}_{k=1}^{\infty}$ is a (monotone) finite dimensional decomposition for its closed linear span $F$ in $\mathscr{P}\left({ }^{n} E\right)$. Since each monomial is a product of continuous linear mappings it follows that $F \subset \mathscr{P}_{\omega}\left({ }^{n} E\right)$. To complete the proof we must show that $\varphi^{n} \in F$ for all $\varphi \in E^{\prime}$.

Let $\varphi \in E^{\prime}$ and let $\psi_{k}=\varphi-\varphi \circ \Pi_{k}=\left.\varphi\right|_{\left\{E_{j}\right\}_{j>k}}$. Since the decomposition is shrinking it follows that $\left\|\psi_{k}\right\| \rightarrow 0$ as $k \rightarrow \infty$. We may suppose that the decomposition $\left\{E_{j}\right\}_{j}$ is monotone. From the identity

$$
a^{n}-b^{n}=(a-b)\left(\sum_{i=0}^{n-1} a^{n-1-i} b^{i}\right)
$$

we see that 


$$
\begin{aligned}
\left\|\varphi^{n}-\left(\varphi \circ \Pi_{k}\right)^{n}\right\| & =\sup _{\|x\| \leq 1}\left|\varphi^{n}(x)-\varphi^{n}\left(\Pi_{k}(x)\right)\right| \\
& \leq \sup _{\|x\| \leq 1}\left|\varphi(x)-\varphi\left(\Pi_{k}(x)\right)\right|\left(\sum_{i=0}^{n-1}|\varphi(x)|^{n-1-i}\left|\varphi\left(\Pi_{k}(x)\right)\right|^{i}\right) \\
& \leq n\left\|\psi_{k}\right\|\|\varphi\|^{n-1} \underset{k \rightarrow \infty}{\longrightarrow} 0 .
\end{aligned}
$$

Hence $\varphi^{n} \in F$ for all $\varphi \in E^{\prime}$ and this completes the proof.

If each $E_{j}$ in proposition 9 is one dimensional then the Banach space $E$ has a shrinking Schauder basis. In general, however, $\mathscr{P}_{k}\left({ }^{n} E\right)$ will not be one dimensional for $n>1$ and we cannot immediately deduce that $\mathscr{P}_{\omega}\left({ }^{n} E\right)$ has a Schauder basis. To obtain this result we need to order a basis in each of the finite dimensional spaces $\mathscr{P}_{k}\left({ }^{n} E\right)$ and in the process of defining this, by induction, we arrive at the square ordering given in $[25,1,13,26]$.

Let $\left(e_{n}\right)_{n=1}^{\infty}$ denote a shrinking monotone basis for $E$. The sequence $\left(e_{n}^{\prime}\right)_{n=1}^{\infty}$ is a basis for $E^{\prime} \cong \mathscr{P}\left({ }^{1} E\right)=\mathscr{P}_{\omega}\left({ }^{1} E\right)$. In monomial terminology this is the sequence of monomials of degree 1 and we denote it by $\left(z_{n}\right)_{n=1}^{\infty}$. This places an order on the basis for $\mathscr{P}\left({ }^{n} E\right)$ when $n=1$. Suppose $\left\{P_{k, j}^{n}\right\}_{j=1}^{\ell_{n}(k)}$ is the ordered basis for $\mathscr{P}_{k}\left({ }^{n} E\right)$ then we define the ordered basis for $\mathscr{P}_{k}\left({ }^{n+1} E\right)$ as

$$
\left\{z_{k} P_{1, j}^{n}\right\}_{j=1}^{\ell_{n}(1)},\left\{z_{k} P_{2, j}^{n}\right\}_{j=1}^{\ell_{n}(2)}, \ldots,\left\{z_{k} P_{k, j}^{n}\right\}_{j=1}^{\ell_{n}(k)}
$$

i.e. $z_{k} P_{r, s}^{n}$ precedes $z_{k} P_{r^{\prime}, s^{\prime}}^{n}$ in the ordering of the basis if either $r<r^{\prime}$ or $r=r^{\prime}$ and $s<s^{\prime}$. We refer to [16] for further details.

Proposition 10. If E has a shrinking Schauder basis then the monomials of degree $n$ with the square order form a Schauder basis for $\left(\mathscr{P}_{\omega}\left({ }^{n} E\right),\|\cdot\|\right)$.

Proof. We may suppose that the basis for $E$ is monotone and normalised. Let $C_{k, n}$ denote the basis constant for $\left\{P_{k, j}^{n}\right\}_{j=1}^{\ell_{n}(k)}$ for all $k$ and $n$. Since $\left\{\mathscr{P}_{k}\left({ }^{n} E\right)\right\}_{k}$ is a finite dimensional decomposition it suffices to show that $\sup _{k} C_{k, n}$ is finite for all $n$. We prove this by induction on $n$. Since the basis for $E$ is shrinking the result is true for $n=1$. We suppose that the result is true for $n$ and that $C_{n}$ is the resulting basis constant for $\mathscr{P}_{\omega}\left({ }^{n} E\right)$.

Let $\left\{P_{k, j}^{n+1}\right\}_{j=1}^{\ell_{n+1}(k)}$ denote the basis for $\mathscr{P}_{k}\left({ }^{n+1} E\right)$. We note that $P_{k, j}^{n+1}=Q_{k, j} \cdot z_{k}$ where $\left\{Q_{k, j}\right\}_{j=1}^{\ell_{n+1}(k)}$ is the basis for $\oplus_{s=1}^{k} \mathscr{P}_{s}\left({ }^{n} E\right)$. Let $1 \leq N<M \leq \ell_{n+1}(k)$ and let $\left(a_{k, j}\right)_{j=1}^{\ell_{n+1}(k)}$ denote an arbitrary sequence of scalars. We have 
BANACH SUBSPACES OF SPACES OF HOLOMORPHIC FUNCTIONS AND... 153

$$
\begin{aligned}
\left\|\sum_{j=1}^{N} a_{k, j} P_{k, j}^{n+1}\right\| & =\left\|\sum_{j=1}^{N} a_{k, j} Q_{k, j} z_{k}\right\| \\
& \leq\left\|\sum_{j=1}^{N} a_{k, j} Q_{k, j}\right\|\left\|z_{k}\right\| \\
& \leq 2\left\|\sum_{j=1}^{N} a_{k, j} Q_{k, j}\right\| \\
& \leq 2 C_{n}\left\|\sum_{j=1}^{M} a_{k, j} Q_{k, j}\right\| .
\end{aligned}
$$

We therefore have to prove that there exists $C^{\prime}>0$ (independent of $k$ and $M)$ such that

$$
\left\|\sum_{j=1}^{M} a_{k, j} Q_{k, j}\right\| \leq C^{\prime}\left\|\sum_{j=1}^{M} a_{k, j} P_{k, j}^{n+1}\right\| .
$$

We have

$$
\begin{aligned}
\left\|\sum_{j=1}^{M} a_{k, j} Q_{k, j}\right\| & =\sup _{\substack{x \in E \\
\|x\| \leq 1}}\left|\sum_{j=1}^{M} a_{k, j} Q_{k, j}(x)\right| \\
& =\sup _{\substack{x=\left[e_{1}, \ldots, e_{k}\right] \\
\|x\| \leq 1}}\left|\sum_{j=1}^{M} a_{k, j} Q_{k, j}(x)\right| \\
& =\left|\sum_{j=1}^{M} a_{k, j} Q_{k, j}(w)\right|
\end{aligned}
$$

for some $w$ of the form $w=\sum_{i=1}^{k} w_{i} e_{i},\|w\|=1$.

If $\left|w_{k}\right|<1 / 2$, let $g(\lambda)=\left(\sum_{j=1}^{M} a_{k, j} Q_{k, j}\right)\left(w+\lambda e_{k}\right)$. The function $g$ is a polynomial over $\mathrm{C}$ and, by the maximum modulus theorem, we have

$$
\sup _{|\lambda|=1}|g(\lambda)| \geq|g(0)|=\left\|\sum_{j=1}^{M} a_{k, j} Q_{k, j}\right\| .
$$

Choose $\lambda,|\lambda|=1$, where the supremum is achieved, and let $\widetilde{w}=w+\lambda e_{k}$. Then

$$
\left|\sum_{j=1}^{M} a_{k, j} Q_{k, j}(\widetilde{w})\right| \geq\left\|\sum_{j=1}^{M} a_{k, j} Q_{k, j}\right\|
$$


and

$$
\|\widetilde{w}\| \leq\|w\|+\left\|\lambda e_{k}\right\| \leq 2
$$

If $\widetilde{w}=\sum_{i=1}^{k} \widetilde{w}_{i} e_{i}$ then

$$
\left|\widetilde{w}_{k}\right|=\left|w_{k}+\lambda\right| \geq|\lambda|-\left|w_{k}\right| \geq 1 / 2 .
$$

If $\left|w_{k}\right| \geq 1 / 2$, let $\widetilde{w}=w$. It is easily checked that $\widetilde{w}$ also satisfies (4.1), (4.2) and (4.3) in this case.

Hence

$$
\left|\sum_{j=1}^{M} a_{k, j} P_{k, j}^{n+1}(\widetilde{w})\right|=\left|\sum_{j=1}^{M} a_{k, j} Q_{k, j}(\widetilde{w})\right| \cdot\left|\widetilde{w}_{k}\right| \geq \frac{1}{2}|| \sum_{j=1}^{M} a_{k, j} Q_{k, j}||
$$

and

$$
\left|\sum_{j=1}^{M} a_{k, j} P_{k, j}^{n+1}(\widetilde{w})\right| \leq\left\|\sum_{j=1}^{M} a_{k, j} P_{k, j}^{n+1}\right\| \cdot\|\widetilde{w}\|^{n+1} \leq 2^{n+1}\left\|\sum_{j=1}^{M} a_{k, j} P_{k, j}^{n+1}\right\| .
$$

Hence

$$
\left\|\sum_{j=1}^{M} a_{k, j} Q_{k, j}\right\| \leq 2^{n+2}\left\|\sum_{j=1}^{M} a_{k, j} P_{k, j}^{n+1}\right\|
$$

and this completes the proof.

In proposition 9 we obtained a monotone finite dimensional decomposition for $\mathscr{P}_{\omega}\left({ }^{n} E\right)$ but in proposition 10 the estimates obtained suggest it is extremely unlikely that the basis with the square ordering is monotone. This important difference allows us to lift the results in proposition 9 to holomorphic functions while the same extension is not possible for the results of proposition 10 .

If $E$ is a Banach space and $\mathscr{U}$ is an open subset of $E$, we let

$$
\begin{aligned}
\mathscr{H}_{\omega}(\mathscr{U})=\{f \in \mathscr{H}(\mathscr{U}): & \text { for all } x \in \mathscr{U} \text { there exists a neighbourhood } W \text { of } x \\
& \text { such that } \left.\left.f\right|_{W} \text { is weakly continuous }\right\},
\end{aligned}
$$

i.e. $\mathscr{H}_{\omega}(\mathscr{U})$ is the space of holomorphic functions on $\mathscr{U}$ which are locally weakly continuous.

If $\left\{E_{j}\right\}_{j}$ is a finite dimensional decomposition for the Banach space $E$ and $A$ is a subset of $E$ we say that $A$ is solid (with respect to the decomposition) if $\Pi_{k}(A) \subset A$ for all $k$. For example, $E$ has a monotone decomposition if and only if the unit ball is solid. Every open set is contained in a solid open set 
and the whole space is solid. If $A$ is a solid open set and $K$ is a compact subset of $A$ then $\overline{\cup_{k} \Pi_{k}(K)}$ is a compact subset of $A$.

An ordering on the set of all pairs $(k, n) \in \mathrm{N}^{2}$ is said to be natural in the first index if $\left(k_{1}, n\right) \leq\left(k_{2}, n\right)$ whenever $k_{1} \leq k_{2}$.

From proposition 9 we obtain the following result.

Proposition 11. If $\left\{E_{j}\right\}_{j}$ is a finite dimensional shrinking Schauder decomposition for the Banach space $E$ and $\mathscr{U}$ is a balanced convex solid open subset of $E$, then $\left\{\mathscr{P}_{k}\left({ }^{n} E\right)\right\}_{k=1, n=0}^{\infty}$ with any ordering which is natural in the first index (k) is a finite dimensional Schauder decomposition for $\left(\mathscr{H}_{\omega}(\mathscr{U}), \tau_{\omega}\right)$.

Proof. We suppose the decomposition is monotone and let $B$ denote the unit ball of $E$. Since the polynomials are dense in $\left(\mathscr{H}(\mathscr{U}), \tau_{\omega}\right)$, proposition 9 shows that $\left\{\mathscr{P}_{k}\left({ }^{n} E\right)\right\}_{k=1, n=0}^{\infty}$ spans a dense subspace of $\left(\mathscr{H}_{\omega}(\mathscr{U}), \tau_{\omega}\right)$. Since $\mathscr{U}$ is solid it contains a fundamental system of compact sets $K$ which are convex and balanced such that $\Pi_{n}(K) \subset K$ for all $K$ and $n$. Hence $E$ normed with the Minkowski functional of $K+\alpha B, \alpha \neq 0$, also has $\left\{E_{n}\right\}_{n}$ as a monotone decomposition.

Let

$$
p\left(\sum_{n=0}^{\infty} \frac{\widehat{d}^{n} f(0)}{n !}\right)=\sum_{n=0}^{\infty}\left\|\frac{\widehat{d}^{n} f(0)}{n !}\right\|_{K+\alpha_{n} B}
$$

for $f \in\left(\mathscr{H}_{\omega}(\mathscr{U}), \tau_{\omega}\right)$. Let $J$ denote a non-empty subset of $\mathrm{N}^{2}$ such that $(k, n) \in J$ implies $\left(k^{\prime}, n\right) \in J$ for $k^{\prime} \leq k$. Let $\left(k_{1}, n_{1}\right) \in \mathrm{N}^{2} \backslash J$ be chosen such that either

$$
\left(k_{1}-1, n_{1}\right) \in J
$$

or

$$
\left(1, n_{1}\right) \notin J .
$$

Let $J^{\prime}=J \cup\left\{\left(k_{1}, n_{1}\right)\right\}$. Let $\left(\alpha_{j}\right)_{j \in J^{\prime}}$ denote an arbitrary set of scalars and let $\left(P_{j}\right)_{j \in J^{\prime}}$ denote a sequence of polynomials with $P_{j} \in \mathscr{P}_{k}\left({ }^{n} E\right)$ if $j=(k, n)$.

If (4.4) holds then 


$$
\begin{aligned}
p\left(\sum_{j \in J} \alpha_{j} P_{j}\right) & =p\left(\sum_{\substack{j \in J \\
j \neq\left(k, n_{1}\right)}} \alpha_{j} P_{j}\right)+p\left(\sum_{\substack{j=\left(k, n_{1}\right) \\
k=1, \ldots, k_{1}-1}} \alpha_{j} P_{j}\right) \\
& \leq p\left(\sum_{\substack{j \in J \\
j \neq\left(k, n_{1}\right)}} \alpha_{j} P_{j}\right)+p\left(\sum_{\substack{j=\left(k, n_{1}\right) \\
k=1, \ldots, k_{1}}} \alpha_{j} P_{j}\right) \quad \text { by proposition } 9 \\
& =p\left(\sum_{j \in J^{\prime}} \alpha_{j} P_{j}\right)
\end{aligned}
$$

If (4.5) holds then

$$
\begin{aligned}
p\left(\sum_{j \in J} \alpha_{j} P_{j}\right) & \leq p\left(\sum_{j \in J} \alpha_{j} P_{j}\right)+p\left(\alpha_{\left(k_{1}, n_{1}\right)} P_{\left(k_{1}, n_{1}\right)}\right) \\
& =p\left(\sum_{j \in J^{\prime}} \alpha_{j} P_{j}\right) .
\end{aligned}
$$

Hence $\left\{\mathscr{P}_{k}\left({ }^{n} E\right)\right\}_{k=1, n=0}^{\infty}$ is a finite dimensional Schauder decomposition for $\left(\mathscr{H}_{\omega}(\mathscr{U}), \tau_{\omega}\right)$.

\section{§5. Reflexivity.}

We now return to the examples which motivated our investigations. In dealing with reflexivity we shall use the following two results;

(5.1) if $E$ is a reflexive Banach space with the approximation property then $\mathscr{P}\left({ }^{n} E\right)=\mathscr{P}_{\omega}\left({ }^{n} E\right)$ if and only if $\left(\mathscr{P}\left({ }^{n} E\right),\|\cdot\|\right)$ is reflexive $([25,2])$,

(5.2) if $E$ is a separable reflexive Banach space with the approximation property and $\mathscr{U}$ is a balanced open subset of $E$ then $\left(\mathscr{H}(\mathscr{U}), \tau_{\omega}\right)$ is reflexive if and only if $\left(\mathscr{P}\left({ }^{n} E\right),\|\cdot\|\right)$ is reflexive for all $n([14])$.

PROPOSITION 12. If $E$ is a reflexive Banach space with a finite dimensional decomposition $\left\{E_{j}\right\}_{j}$ then the following are equivalent:

(a) $\left(\mathscr{P}\left({ }^{n} E\right),\|\cdot\|\right)$ is reflexive for all $n$,

(b) $\left\{\mathscr{P}_{k}\left({ }^{n} E\right)\right\}_{k=1}^{\infty}$ is a finite dimensional Schauder decomposition for $\mathscr{P}\left({ }^{n} E\right)$, for all $n$,

(c) $\left\{\mathscr{P}_{k}\left({ }^{n} E\right)\right\}_{k=1, n=0}^{\infty}$, with the order described in proposition 11 , is a finite dimensional Schauder decomposition for $\left(\mathscr{H}(\mathscr{U}), \tau_{\omega}\right)$ for any solid open subset $\mathscr{U}$ of $E$,

(d) $\left(\mathscr{H}(\mathscr{U}), \tau_{\omega}\right)$ is reflexive for any balanced open subset $\mathscr{U}$ of $E$, 
(e) $\mathscr{H}(\mathscr{U})=\mathscr{H}_{\omega}(\mathscr{U})$ for any open subset $\mathscr{U}$ of $E$.

Proof. Since $E$ is reflexive the decomposition $\left\{E_{j}\right\}_{j}$ is shrinking and $E$ has the approximation property. Using Taylor series expansions we see that $\mathscr{H}(\mathscr{U})=\mathscr{H}_{\omega}(\mathscr{U})$ for arbitrary open subsets of $E$ if, and only if, $\mathscr{P}\left({ }^{n} E\right)=\mathscr{P}_{\omega}\left({ }^{n} E\right)$ for all $n$. Hence, by $(5.1),(\mathrm{a}) \Leftrightarrow(\mathrm{e})$, and, by $(5.2),(\mathrm{a}) \Leftrightarrow(\mathrm{d})$. By proposition 9 and (5.1) we have (a) $\Leftrightarrow$ (b) and, by proposition 11 , $(\mathrm{c}) \Leftrightarrow(\mathrm{e})$. This completes the proof.

Proposition 10 and (5.1) recovers the result (1.2) of Alencar.

Finally we return to our original motivation (1.1). We have the following theorem which leads to an answer to our original problem.

THEOREM 13. If $\left\{E_{j}\right\}_{j}$ is a shrinking finite dimensional unconditional Schauder decomposition for the Banach space $E$ then the following are equivalent:

(1) $\mathscr{P}_{\omega}\left({ }^{n} E\right)=\mathscr{P}\left({ }^{n} E\right)$ for all $n$,

(2) $\left\{\mathscr{P}_{k}\left({ }^{n} E\right)\right\}_{k=1}^{\infty}$ is a finite dimensional decomposition for $\mathscr{P}\left({ }^{n} E\right)$, for all $n$,

(3) $\left(\mathscr{P}\left({ }^{n} E\right),\|\cdot\|\right)$ is separable for all $n$,

(4) $c_{0} \nrightarrow \mathscr{P}\left({ }^{n} E\right)$ for any positive integer $n$,

(5) $c_{0} \nrightarrow\left(\mathscr{H}(E), \tau_{\omega}\right)$.

Proof. We may suppose, without loss of generality, that the unconditionality constant of the decomposition is 1 . By corollary $5,(4) \Leftrightarrow(5)$. By proposition $9,(1) \Leftrightarrow(2)$. Clearly $(2) \Rightarrow(3)$ and since $\left(\mathscr{P}\left({ }^{n} E\right),\|\cdot\|\right)$ is a dual space $(3) \Rightarrow(4)$. To complete the proof we show that $(4) \Rightarrow(1)$.

We suppose that (1) holds for all $l<n$ but that (1) does not hold for $n$. Clearly we must have $n \geq 2$. Hence there exists $P \in \mathscr{P}\left({ }^{n} E\right)$ which is not weakly continuous on bounded sets. Since $E^{\prime}$ has a finite dimensional decomposition it is separable and hence the weak topology on bounded subsets of $E$ is metrizable. We may therefore suppose that $P$ is not weakly sequentially continuous and by using the binomial theorem we see, as in [2], that $P$ is not weakly sequentially continuous at the origin. Hence there exists a weakly null sequence $\left(x_{k}\right)_{k}$ and $\varepsilon>0$ such that $\left|P\left(x_{k}\right)\right| \geq \varepsilon$ for all $k$. We now fix an arbitrary positive integer $l$. Let $y_{k}=\Pi_{l}\left(x_{k}\right)$ and $z_{k}=x_{k}-\Pi_{l}\left(x_{k}\right)$ for all $k$. We have $\left\|y_{k}\right\| \rightarrow 0$ as $k \rightarrow \infty$ and $\left(z_{k}\right)_{k}$ is a weakly null sequence in $E$.

Since $\sum_{j=1}^{l} E_{j}$ is finite dimensional it has a Schauder basis $e_{1}, \ldots, e_{s}$. If $F_{i}$ is the one dimensional subspace of $E$ spanned by $e_{i}, 1 \leq i \leq s$, and $F_{s+1}$ denotes the closed subspace spanned by $\left\{E_{j}: j>l\right\}$ then $\left\{F_{i}\right\}_{i=1}^{s+1}$ is a Schauder decomposition for $E$. Since it is a decomposition into a finite number of subspaces it is an unconditional shrinking, not necessarily monotone, Schauder decomposition. If we now take the monomial expansion of $P$ with respect to this decomposition, we obtain 


$$
P\left(\sum_{i=1}^{s} \lambda_{i} e_{i}+x\right)=P(x)+\sum_{\substack{m \in N^{s} \\ 0<|m|<n}} a_{m} \lambda^{m} Q_{m}(x)+Q_{0}\left(\lambda_{1}, \ldots, \lambda_{s}\right)
$$

where $\lambda_{i} \in \mathrm{C}, x \in F_{s+1}, Q_{m} \in \mathscr{P}\left({ }^{n-|m|} F_{s+1}\right), a_{m} \in \mathrm{C}$ and $Q_{0}$ is $P$ restricted to $\sum_{j=1}^{l} E_{j}$.

Since $Q_{m} \in \mathscr{P}\left({ }^{n-|m|} F_{s+1}\right)$ with $|m|>0$ it follows by hypothesis that $Q_{m}\left(z_{k}\right) \rightarrow 0$ as $k \rightarrow \infty$. Hence $\left|P\left(z_{k}\right)\right| \nrightarrow 0$ as $k \rightarrow \infty$.

Using the Schauder decomposition and a diagonal process we can generate a disjointly supported bounded sequence of vectors $\left(w_{k}\right)_{k}$, such that $\left|P\left(w_{k}\right)\right| \geq \varepsilon$ for all $k$. Thus, there exists a strictly increasing sequence of positive integers $\left(n_{k}\right)_{k}$ such that $w_{k}=\sum_{j=n_{k}+1}^{n_{k+1}} v_{j}$ for all $k$, where $v_{j} \in E_{j}$ for all $j$.

For each integer $k$, we let $P_{k}=P \circ\left(\Pi_{n_{k+1}}-\Pi_{n_{k}}\right)$. We have $\left\|P_{k}\right\| \leq\|P\|$ $\left\|\Pi_{n_{k+1}}-\Pi_{n_{k}}\right\|^{n} \leq 2^{n}\|P\|$. Hence $\left(P_{k}\right)_{k}$ is a bounded sequence in $\left(\mathscr{P}\left({ }^{n} E\right),\|\cdot\|\right)$. By our construction we have

$$
\left|P_{k}\left(w_{k}\right)\right|=\left|P\left(w_{k}\right)\right| \geq \varepsilon \quad \forall k .
$$

If $x=\sum_{j=1}^{\infty} x_{j} \in E, x_{j} \in E_{j}$ for all $j$, then for all positive integers $l$ we have

$$
\begin{aligned}
\sum_{k=1}^{l}\left|P_{k}(x)\right| & =\sum_{k=1}^{l}\left|P\left(\sum_{j=n_{k}+1}^{n_{k+1}} x_{j}\right)\right| \\
& \leq \sup _{\left|\lambda_{k}\right| \leq 1}\left|P\left(\sum_{k=1}^{l} \lambda_{k}\left(\sum_{j=n_{k}+1}^{n_{k+1}} x_{j}\right)\right)\right| \leq 2^{n}\|P\|\|x\|^{n}
\end{aligned}
$$

by unconditionality.

Since $\left(P_{k}\right)_{k}$ is not a null sequence in $\mathscr{P}\left({ }^{n} E\right),[18$, theorem 2] implies that $c_{0} \hookrightarrow \mathscr{P}\left({ }^{n} E\right)$. Hence $(4) \Rightarrow(1)$ and this completes the proof.

REMARK 14. It is rather easy to add extra conditions in theorem 13 and we briefly mention some possibilities in this direction.

(a) Using $\mathscr{S}$-Schauder decompositions it is easy to see that condition (3) is equivalent to the separability of $\left(\mathscr{H}(\mathscr{U}), \tau_{\omega}\right)$, for any balanced open subset $\mathscr{U}$ of $E$ (in fact this equivalence is true for any Banach space).

(b) If $E$ has a shrinking Schauder basis then, by proposition 10, condition (2) is equivalent to the condition that the monomials, with the square order, are a Schauder basis for $\mathscr{P}\left({ }^{n} E\right)$ for all $n$. The equivalence of (2) and (4) gives (1.1) for homogeneous polynomials.

(c) If we assume in addition that $E$ is reflexive in theorem 13, then proposition 12 shows that the conditions in theorem 13 are equivalent to the condition that $\left(\mathscr{P}\left({ }^{n} E\right),\|\cdot\|\right)$ is reflexive for all $n$. We note in passing that reflexivity always implies the property "does not contain $c_{0}$ " but for spaces of 
polynomials (or holomorphic functions) the unconditionality hypothesis implies that they are equivalent.

A disjointly supported sequence of vectors $\left(x_{k}\right)_{k=1}^{\infty}$ in a Banach space $E$ with a finite dimensional decomposition $\left\{E_{j}\right\}_{j}$ has a lower $q$ estimate, $1<q<\infty$, if there exists $C>0$ such that for any sequence of scalars $\left(\lambda_{k}\right)_{k=1}^{\infty}$ and for any $l \in \mathrm{N}$ we have

$$
\left\|\sum_{k=1}^{l} \lambda_{k} x_{k}\right\|^{q} \geq C \sum_{k=1}^{l}\left\|\lambda_{k} x_{k}\right\|^{q} .
$$

In the proof of the implication (4) $\Rightarrow(1)$ of theorem 13, we see that if $\mathscr{P}_{\omega}\left({ }^{n} E\right) \neq \mathscr{P}\left({ }^{n} E\right)$ for some $n$ then there exists a disjointly supported sequence of unit vectors $\left(w_{k}\right)_{k=1}^{\infty}$ and $P \in \mathscr{P}\left({ }^{n} E\right)$ such that $\left|P\left(w_{k}\right)\right| \geq \varepsilon$ for all $k$. The proof of this result did not use unconditionality. We can therefore apply theorem II.4.4 of [21] to obtain the following.

Proposition 15. If $\left\{E_{j}\right\}_{j}$ is a shrinking finite dimensional Schauder decomposition for the Banach space $E$ and no disjointly supported sequence of vectors in $E$ has a lower q estimate, $1<q<\infty$, then $\mathscr{P}_{\omega}\left({ }^{n} E\right)=\mathscr{P}\left({ }^{n} E\right)$ for all $n$.

Proposition 15 applies, for instance, to $c_{0} \times T^{\prime}$ and $T_{J}^{\prime}$.

\section{REFERENCES}

1. R. Alencar, On reflexivity and basis for $\mathscr{P}\left({ }^{m} E\right)$, Proc. Royal Irish Acad. 85A (1985), 1-8.

2. A. Alencar, R. Aron and S. Dineen, A reflexive space of holomorphic functions in infinitely many variables, Proc. Amer. Math. Soc. (1984), 407-411.

3. J. M. Ansemil and S. Ponte, The compact open and the Nachbin ported topologies on spaces of holomorphic functions, Arch. Math. 51 (1988), 65-70.

4. R. Aron, Compact polynomials and compact differentiable mappings between Banach spaces, Seminaire P. Lelong, Lecture Notes in Math. 524 (1976), 2-222.

5. R. Aron and S. Dineen, Q-reflexive Banach spaces, Rocky Mountain J. Math. (to appear)

6. R. Aron and J. Globevnik, Analytic functions on $c_{0}$, Revista Matemática de la Univ. Complutense de Madrid 2 (1989), 27-33.

7. R. Aron, C. Hervés and M. Valdivia, Weakly continuous mappings on Banach spaces, J. Funct. Anal. 52 (1983), 189-204.

8. R. Aron and J. Prolla, Polynomial approximation of differentiable functions on Banach spaces, J. Reine Angew. Math. 3 (1980), 195-216.

9. J. Bonet and A. Peris, On the injective tensor product of quasinormable spaces, Results Math. 20 (1991), 431-443.

10. A. Defant and M. Maestre, Property (BB) and holomorphic functions on Fréchet- Montel spaces, Math. Proc. Cambridge Philos. Soc. 115 (1993), 303-313.

11. J. B. Díaz, On 2-homogeneous polynomials on some non-stable Banach and Fréchet spaces, Studia Math. 126 (3) (1997), 291-307.

12. J. Diestel, Sequences and series in Banach spaces, Graduate Texts in Math. 92, 1984.

13. V. Dimant and I. Zalduendo, Bases in spaces of multilinear forms over Banach spaces, J. Math. Anal. Appl. 200 (1996), 548-566. 
14. S. Dineen, Complex analysis in locally convex spaces, North Holland Math. Studies 57 (1981).

15. S. Dineen, A Dvoretzky theorem for polynomials, Proc. Amer. Math. Soc. 123 (1995), 28172821.

16. S. Dineen, Complex Analysis on Infinite Dimensional Spaces, Book in preparation.

17. S. Dineen, Holomorphic Functions on Fréchet spaces with Schauder basis, Extracta Math. 10 (1995), 164-167.

18. S. Dineen and M. Lindström, Spaces of homogeneous polynomials containing $c_{0}$ or $\ell^{\infty}$, Proc. First Trier Workshop on Fréchet spaces (1994), Functional analysis, Eds. Dierolf, Dineen, Dománski, Walter de Gruyter, Berlin 1996, 119-127.

19. J. D. Farmer, Polynomial reflexivity in Banach spaces, Israel J. Math. 87 (1994), 257-273.

20. J. Gómez and J. A. Jaramillo, Interpolation by weakly differentiable functions on Banach spaces, J. Math. Anal. Appl. 182, 2 (1994), 501-515.

21. R. Gonzalo, Smoothness and Polynomials on Banach spaces, Doctoral Dissertation, Universidad Complutense, Madrid, 1994.

22. R. Gonzalo and J. A. Jaramillo, Compact polynomials on Banach spaces, Extracta Math. 8 (1993), 42-48.

23. R. Gonzalo and J. A. Jaramillo, Smoothness and estimates of sequences in Banach spaces, Israel J. Math. 89 (1995), 312-341.

24. J. Lindenstrauss and L. Tzafriri, Classical Banach spaces, Vol. 1, Sequence Spaces, SpringerVerlag, 1977.

25. R. Ryan, Applications of topological tensor products to infinite dimensional holomorphy, $\mathrm{Ph}$. D. thesis, Trinity College, Dublin, 1980.

26. R. Ryan, The Dunford-Pettis property and projective tensor products, Bull. Polish Acad. Sci. Math. 35, No. 11-12 (1987), 785-792.

27. I. Zalduendo, An estimate for multilinear forms on $\ell^{p}$ spaces, Proc. Roy. Irish. Acad. Sect. A 93 (1993), 7-142.

DEPARTAMENTO DE ECONOMÍA Y MATEMÁTICA

UNIVERSIDAD DE SAN ANDRÉS

VITO DUMAS 284

(1644) VICTORIA

PROV. DE BUENOS AIRES

ARGENTINA
DEPARTMENT OF MATHEMATICS UNIVERSITY COLLEGE DUBLIN

BELFIELD

DUBLIN 4

IRELAND 